\title{
APPLYING VIRTUAL PROTOTYPING TO THE INNOVATIVE DESIGN OF LOW ENERGY ACCELERATORS*
}

\author{
Mingwu Fan, Shiqi Li, Tiaoqin Yu, Dezhi Chen, Yongqian Xiong \\ Huazhong University of Science and Technology, Wuhan 430074, CHINA
}

\begin{abstract}
Based on the accelerator theory and virtual prototyping technique, an accelerator virtual prototyping system (AVPS) is formulated, implemented for the innovative design of low energy accelerators. The framework of AVPS takes into account the needs of manufacture, assembly/disassembly, operation, and maintenance in a virtual environment during the design phase. In order to guarantee the reliability of AVPS, Augmented Reality (AR) technique is used to integrate the virtual environment and experimental subsystem. Common Object Request Broker Architecture (CORBA) middleware for distributed, object-oriented applications is used to develop and implement the framework. The main components of AVPS include AR subsystem, distributed computing environment and data warehouse.
\end{abstract}

\section{INTRODUCTION}

In 1990s, with the development of Virtual Reality (VR) and $\mathrm{CAD} / \mathrm{CAM} / \mathrm{CAE}$ technologies, a new design method called Virtual Prototyping (VP) has been proposed and used in design of airplanes, cars and other mechanical products. Comparing with $\mathrm{CAD} / \mathrm{CAM} / \mathrm{CAE}$ methods, VP presents an integrated design environment in which the design, manufacture, assembly, operation and maintenance could be discussed before the machine being built. Recur to the use of VR technology, VP provides a friendly interactive interface which allows designers to track and to control the design process. In other words, VP is a development process completely implemented in computer, which can be used to replace or decrease the physical experimental prototype. Consequently, VP is an ideal method to condense time, costless, and risk of product development.

In the field of accelerators, CERN used VR to guide the design of LHC in 1996, and a Virtual Environment Navigation in the Underground Sites system (VENUS) was developed. Using VENUS, engineers can easily to know the work process of LHC and its complex structure. Subsequently, the concept of "virtual accelerators" was proposed by KEK and Jefferson Lab [1-3].

This paper applied VP technique into the research of low energy accelerators. The unique of VP we used is based on augmented reality (AR), which integrates the computing subsystem and experimental subsystem together in the interactive environment. After proposing the framework of AVPS, the key techniques are discussed. A design example of a $30 \mathrm{MeV}$ cyclotron is given to show how AVPS system works.

\footnotetext{
* Supported by National Nature Science Foundation of China
}

\section{FRAMEWORK OF AVPS}

The hiberarchy of AVPS developed by HUST has four layers, namely, the interface layer, the application layer, the data layer, and the support layer, as shown in Figure 1. The interface layer is an augmented reality subsystem made up of virtual environment and experimental subsystem. By using of sensors and actuators, users can interact with AVPS in a natural way. The application layer is a distributed computing environment (DCE) based on CORBA. Programs used for modelling, analysis, manufacture, assembly, debugging and maintenance are encapsulated as a CORBA object. The data layer forms a data warehouse made up of database, knowledge base and corresponding management programs. It provides data information, status information and control information of the system, including the accelerator's performance data, geometry data, and engineering data produced in the process of evolving. The technique standards, criterion and generic data used in the whole produce period are stored in the knowledge base. The support layer is the basis of the AVPS, it includes computer operating system (OS), network, protocol, database management system and knowledge base management system.

\section{TECHNIQUE CHARACTERISTICS}

\section{Augmented Reality}

As a bridge linking the real object and the virtual reality, the basic idea of an AR system is the mixing of real and virtual images with an enhancement of the user's perceptions of the real world. AR integrates VR environment and real world into an entity, in which they can complement each other. In AVPS, the VR environment is an advanced interface through which users can design, analyze, manufacture, assembly/disassembly, and maintain accelerators in a natural way. Considering that AVPS needs high performance graphics display ability, OpenGL and Performer are used as a develop tool to program the VR environment on SGI graphics workstation. Then CORBA is used to link it with other components. The other important part in the AR system is the experimental system.

\section{Distributed Computing Environment}

CORBA has been selected as middleware for building a distributed computing environment, which is a software standard for distributed, heterogeneous, object-oriented applications. It is open, robust, interoperable, multiplatform and multi-user supported. CORBA applications are composed of objects, individual units of running 
software that combine functionality and data, and that frequently represent something in the real world. The interface is the syntax part of the contract that the server object offers to the clients invoking it. Any client that wants to invoke an operation on the object must use this IDL interface to specify the operation, and to marshal the arguments that it sends. When the invocation reaches the target object, the same interface definition is used to unmarshal the arguments so that the object can perform the requested operation with them. The interface definition is then used to marshal the results for their trip back, and to unmarshal them when they reach their destination. In AVPS, a typical client and object implementation is virtual reality environment and analysis component such as magnet, cavity, injection et al.

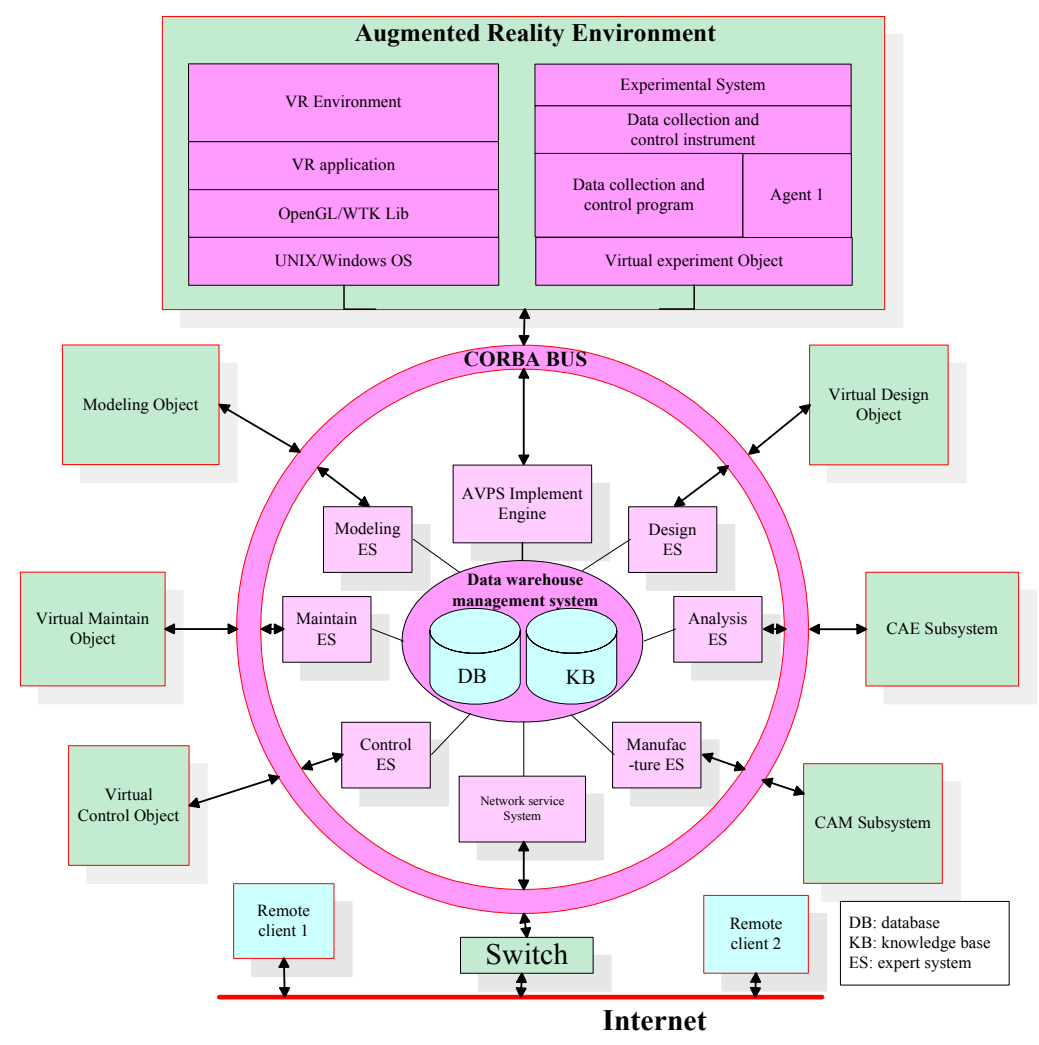

Figure 1: Framework of AVPS

\section{Data Warehouse}

The essential of the AVPS is to use an exact digital model to simulate all activities of an accelerator during its lifecycle. Therefore, an efficacious data warehouse is very important. It must include the following models: the structural data, physical property data, environment data, accelerator operation data, interlock and corresponding management system. All these models are integrated into a database and knowledge base. In order to manage and access the complex information in AVPS, data warehousing approach is used, in which the integrated information is available for immediate querying and analysis by clients.

\section{APPLYING AVPS TO SIMULATE THE EVOLVING PROCESS OF ACCELERATORS}

AVPS is an integrated solution scheme of accelerator's design, analysis, and simulation of manufacture, assembly, controlling, and maintaining. Through AVPS, engineers can simulate accelerators by using coarse granularity models or refined ones, can test the whole equipment or its subsystem in computer. The fidelity that AVPS can get is decided by the granularities of AVPS model. A refined prototype model can simulate accelerators realistically. It describes accelerator and its subsystem in detail. Fig.2 shows the prototype model of a $30 \mathrm{MeV}$ accelerator, CYCIAE-30.

\section{Modelling the Accelerator}

Modelling is the base of virtual prototyping. Its purpose is to create virtual prototype of the accelerator. The virtual prototype should include all the geometrical and physical information, such as the geometry shape, the positions and link relations, and physical properties of the parts, and beam information. Management of virtual prototype is the first considerable subject in this stage. Three model libraries, namely, basic library, auxiliary library and custom library, can be built. The basic library stores basic information such as geometry shape, the 
position and link relation of the parts. While the auxiliary library stores physical properties, particle beam information, and so on. The custom library is designed for the user's expending.

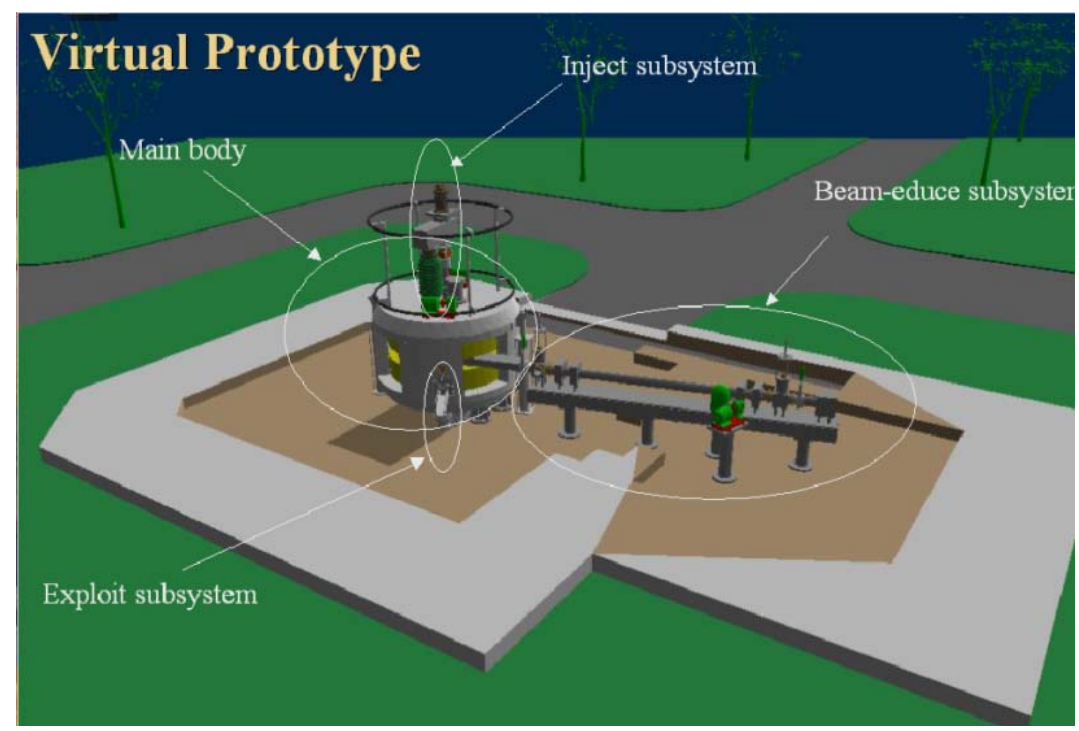

Figure 2: Virtual Prototype of CYCIAE-30 Created by AVPS

Another important subject is the structure of virtual prototype. In AVPS, both graph structure and tree structure methods are used to represent virtual prototype. The relationships among accelerator parts are represented by graph structure method. While the tree structure method is used to describe the hiberarchy of accelerator. In AVPS, engineers can build basic elements firstly, and then organize them into a higher layer, and integrate layers into a uniform system at last.

\section{Virtual Assembly/Disassembly}

Virtual assembly/disassembly is the digital mapping of real corresponding process. In order to optimize the path and process of assembly/disassembly, process programing and collision detection technology must be used to handle virtual prototypes created in the modeling module.

The assembly module of AVPS is an interactive, 3D graphic simulation tool, specifically designed for use in developing and documenting assembly processes. Apart from simulating complex assembly/disassembly processes, users can also investigate the assembly feasibility, determine required product design changes, document optimal assembly sequences, and estimate the cycle time involved, with the help of sequence charts. These can also be extended to produce training "movies" for shop floor personnel. Some of the main features of this module are:

- Interactive creation of part trajectory paths.

- Automatic creation of collision-free trajectory paths.

- Interactive creation of process sequences displayed by sequence charts.

- Sequence output in standard spreadsheet formats for integration into assembly process plan documentation.

- A single-pick REVERSE function, which reverses an existing assembly sequence into a disassembly sequence and vice versa.

\section{Particle Motion Simulation}

Based on the analysis of main parts, simulation of the accelerator and its subsystems can be carried out in AVPS. These subsystems include the particle injecting system, the accelerating system, the vacuum control system, and the cooling control system, and so on. Integrating parts analysis, subsystem simulation, and model library, AVPS provide the function to simulate the working process and also, AVPS can help engineer to simulate the process of manufacture, control, cooling, and maintain, and change correspond parameters to optimize its performance.

\section{CONCLUSION}

A CORBA based virtual prototyping system, AVPS, was proposed, implemented, and used to analysis, design and simulate the evolving process of low energy accelerators. It integrates experimental system, distributed numerical simulation system, and virtual reality environment into a uniform framework. Therefore, AVPS can be applied to condense time, and risk, and to design new type of accelerator.

\section{REFERENCES}

[1] J. F. Balaguer, de Gennaro Silvano, "VENUS: A virtual reality project at CERN", Computer Graphics (ACM), 1994, 30(4): 40-43

[2] K. Noriichi, "Virtual accelerator and fundamental guidelines towards sharable software for accelerator control systems", Nuclear Instruments \& Methods in Physics Research, 1994, Elsevier Science B V: 497-500

[3] J. L. Anderson, "Technology development for the accelerator production of Tritium", Proc. of PAC 1999, March 27-April 2, New York, v1: 571-573 